\title{
Src/STAT3 signaling pathways are involved in KAI1-induced downregulation of VEGF-C expression in pancreatic cancer
}

\author{
XU LIU, XIAOZHONG GUO, HONGYU LI, JIANG CHEN and XINGSHUN QI
}

Department of Gastroenterology, General Hospital of Shenyang Military Area, Shenyang, Liaoning 110000, P.R. China

Received March 30, 2015; Accepted January 28, 2016

DOI: $10.3892 / \mathrm{mmr} .2016 .5093$

\begin{abstract}
A previous study by our group demonstrated that overexpression of KAI1 was associated with lymphatic metastasis in pancreatic cancer. The present study further investigated the signaling pathways involved in KAI1-induced downregulation of vascular endothelial growth factor $\mathrm{C}$ (VEGF-C) and lymphatic metastasis in pancreatic cancer. Immunohistochemistry was performed to examine KAI1 and VEGF-C expression in 28 surgically resected pancreatic cancer tissues. MIA PaCa-2 and PCAN1 pancreatic cancer cell lines were transfected with KAI1 overexpression vector. VEGF-C expression as well as phosphorylation of Src and signal transducer and activator of transcription (STAT)3 were assessed by western blot analysis. Furthermore, the signal transduction inhibitors PP2 and AG490 were used to block the Src and STAT3 signaling pathways, respectively. KAI1 was negatively correlated with VEGF-C expression in pancreatic tumor samples. In MIA PaCa-2 cells, VEGF-C expression was more significantly inhibited by restoration of KAI1 than that in PCAN1 cells. In addition, Src and STAT3 phosphorylation was decreased by KAI1 in MIA PaCa-2 cells. Of note, pre-treatment with PP2 efficiently reversed the KAI1-induced enhancement of Src and STAT3 phosphorylation as well as VEGF-C expression. Pre-treatment with AG490 efficiently reversed the KAI1-induced enhancement of STAT3 phosphorylation and VEGF-C expression, but had no effect on the upregulation of Src phosphorylation. The present study identified the involvement of Src/STAT3 signaling pathways in KAI1-induced downregulation of VEGF-C expression and suggested the implication of these pathways in lymphatic metastasis of pancreatic cancer.
\end{abstract}

Correspondence to: Dr Xiaozhong Guo, Department of Gastroenterology, General Hospital of Shenyang Military Area, 83 Wenhua Road, Shenyang, Liaoning 110000, P.R. China

E-mail: guoxiaozhong1962@163.com

Key words: KAI1, vascular endothelial growth factor C, Src, signal transducer and activator of transcription 3 , pancreatic cancer

\section{Introduction}

Lymph node metastasis is a hallmark of pancreatic cancer (PC) progression and represents one of the most important prognostic factors. The formation of new lymphatic vessels, known as lymphangiogenesis, has an important role in this process (1), although the molecular mechanisms largely remain to be elucidated. Vascular endothelial growth factor $\mathrm{C}$ (VEGF-C) is the central regulator of lymphangiogenesis, and increased expression in various types of primary tumor is correlated with the capacity of tumor cells to disseminate to regional lymph nodes $(2,3)$. Implantation of cells overexpressing VEGF-C has been shown to induce tumor-associated lymphangiogenesis in several orthotopic transgenic mouse models $(4,5)$. In addition, anti-VEGF-C treatment has been shown to inhibit VEGF-C-induced lymphatic hyperplasia and tumor cell migration to draining lymph nodes (6).

KAI1, also known as CD82, is a metastasis-suppressor gene belonging to the tetraspanin family (7). The protein has four hydrophobic transmembrane domains, two extracellular domains and three short intracellular domains. Downregulation of KAl1 expression is often observed in advanced stages of several types of human cancer. Loss of KAI1 expression has been shown to be associated with increased cell migration, reduced homotypic cell adhesion and altered ability of tumor cells to bind specific extracellular proteins (8). These changes result in increased invasiveness of tumor cells in vitro as well as a tendency to metastasize in vivo; thus, the KAI1 gene is implicated in the suppression of lymphatic metastasis.

In a previous study by our group, KAI1 was found to be able to reduce lymphangiogenesis, as indicated by detection of lymphatic vessel endothelial hyaluronan receptor-1 (9). Increased VEGF-C expression has also been demonstrated to be closely associated with lymphangiogenesis in PC invasion and lymphatic metastasis (10). Therefore, the present study examined the association between KAI1 and VEGF-C expression and investigated the signaling pathways involved in the KAI1-induced decreases in VEGF-C expression and lymphatic metastasis the MIA PaCa-2 and PANC1 PC cell lines.

\section{Materials and methods}

Tumor samples and immunohistochemistry. Tumor samples were obtained from 28 patients with PC who were treated at the General Hospital of Shenyang Military Area (Shenyang, 
China) from September 2010 to September 2013. All of the patients underwent local tumor resection and synchronal abdomen dissection. The present study was approved by the ethics committee of the General hospital of Shenyang Military Area (Shenyang, China). Informed consent was obtained from all patients. Lymph node (LN) metastases were found in 23 patients $(82 \%)$, while seven patients (25\%) had metastases limited to node level (NL)1 (i.e., peripancreatic nodes). Metastases up to NL2 (nodes along main arteries and the hepatic hilum) and NL3 (pre-aortic nodes) were found in six (21\%) and $10(36 \%)$ patients, respectively, according to the diagnostic criteria of the Japan Pancreas Society (11).

Immunohistochemistry. Paraffin-embedded tumor tissue sections $(4 \mu \mathrm{m})$ were dewaxed and rehydrated. KAIl and VEGF-C were detected using specific primary antibodies [KAI1 (1:100 dilution; BD Pharmingen, Heidelberg, Germany; VEGF-C (1:50 dilution; Zytomed, Berlin, Germany]. The avidin-biotin technique was applied with diaminobenzidine (BIOSS, Beijing, China) as the substrate for visualization of KAI1 and VEGF-C immunoreactivity, which was graded based on the intensity and the percentage of positive cells. Hematoxylin (BIOSS) was used for counterstaining. The staining intensity was scored from 0 to 3 ( $0=$ no staining; 1 = weak, 2 = moderate and 3 = strong staining intensity) using an inverted fluorescent microscope (CKX41-A32PH, Olympus Corporation, Tokyo, Japan). The evaluation and scoring of immunohistochemically stained slides were carried out by 2 independent researchers

Cell culture and transfection. The MIA PaCa-2 and PANC1 human PC cell lines were provided by the Shanghai Institute of Cell Biology, Chinese Academy of Sciences (Shanghai, China). The cells were grown as sub-confluent monolayers in Dulbecco's modified Eagle's medium (DMEM; GE Healthcare Life Sciences, Logan, UT, USA) supplemented with $10 \%$ fetal calf serum (FCS; GE Healthcare Life Sciences), $2 \mathrm{mmol} / 1$ L-glutamine, $100 \mathrm{IU} / \mathrm{ml}$ penicillin $\mathrm{G}$, and $100 \mu \mathrm{g} / \mathrm{ml}$ streptomycin at $37^{\circ} \mathrm{C}$ in a humidified atmosphere containing $5 \% \mathrm{CO}_{2}$. KAI1 overexpression plasmid (pCMV-KAI1 DNA) was a kind gift from Dr Jin-Tang Dong (Emory University School of Medicine, Atlanta, Georgia, USA). Plasmid transfection was performed according to a protocol of a previous study by our group (9). The pCMV-KAI1-transfected cells were renamed as MIA PaCa-2-K and PANC-1-K. The cells transfected with empty pCMV vector (BIOSS) were renamed as MIA PaCa-2-p and PANC-1-p.

For the inhibition experiments, $1.0 \times 10^{6}$ cells were plated in $10-\mathrm{cm}$ dishes and maintained in DMEM with $10 \%$ FCS. When the cells reached $70-80 \%$ confluence, the cell culture medium was replaced with serum-free minimum essential medium (5 ml MEM; Gibco; Thermo Fisher Scientific, Inc., Waltham, MA, USA) containing the $100 \mu \mathrm{M}$ Src inhibitor (incubated for 48 h; PP2; EMD Millipore, San Diego, CA, USA) and $100 \mu \mathrm{M}$ STAT3 inhibitor (incubated for 16 h; AG490; EMD Millipore). Cell lysates were harvested after $24 \mathrm{~h}$ for western blot analysis.

Western blot analysis. Cells were harvested in a lysis buffer obtained from Biogoodland (Wuxi, China) (10 mmol/l

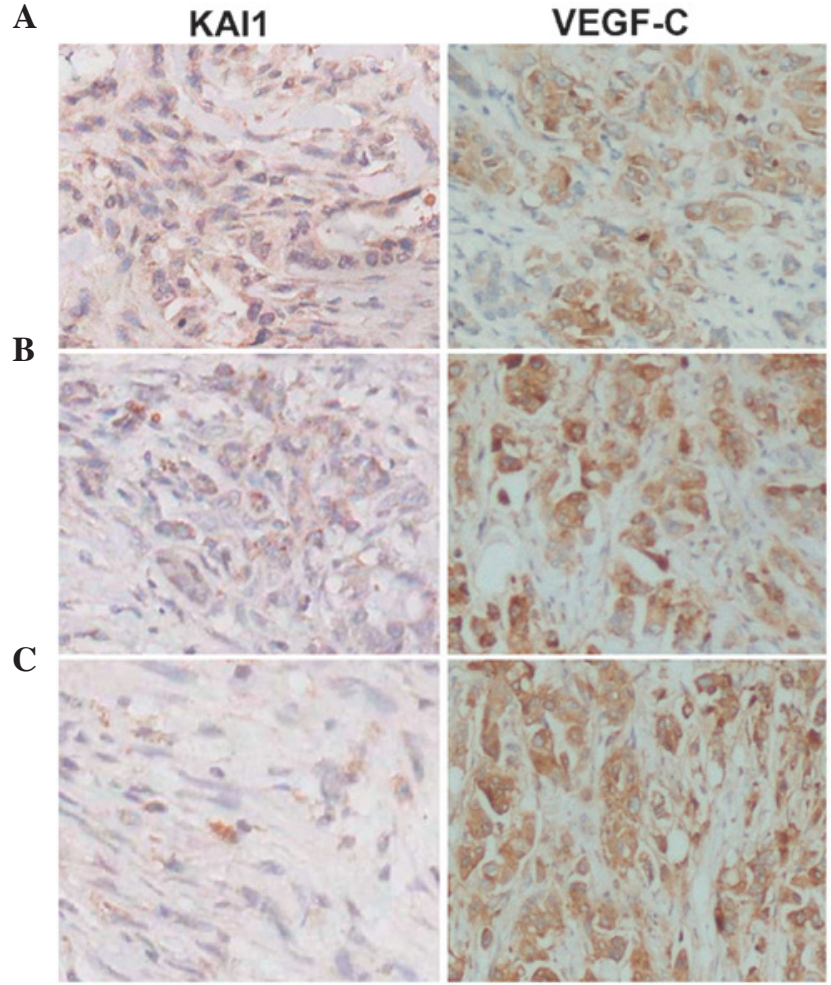

Figure 1. KAI1 and VEGF-C expression in pancreatic tumor samples. (A) Tumor samples without lymph node metastasis. (B) Tumor samples with node metastasis to NL1. (C) Tumor samples with node metastasis to NL3. DAB (brown) was used as a chromogen (magnification, x400). Samples were counterstained with hematoxylin. VEGF, vascular endothelial growth factor; NL node level.

A

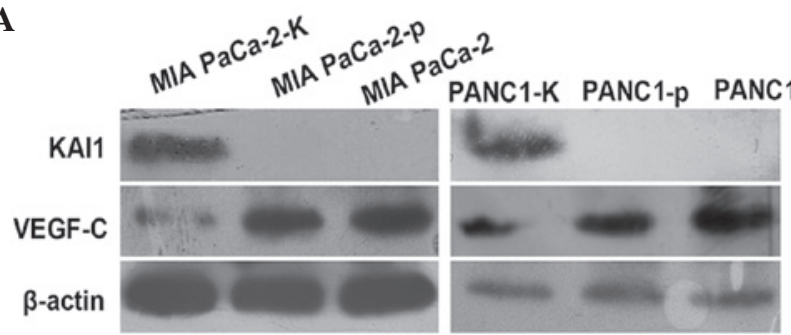

B

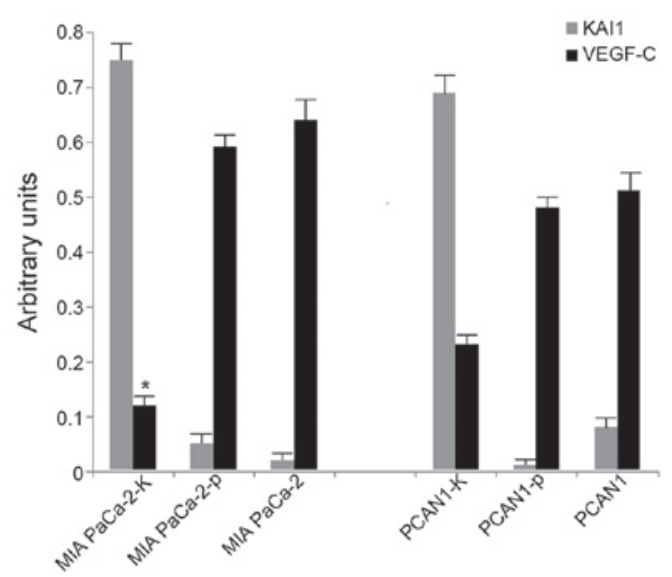

Figure 2. KAI1 decreases the protein expression of VEGF-C in MIA PaCa-2 and PCAN1 cells. (A) Representative western blots of VEGF-C and KAI1 expression. (B) Quantification of protein expression by densitometric analysis. Values are expressed as the mean \pm standard deviation from three or four independent experiments ${ }^{*} \mathrm{P}<0.05$ vs. control. VEGF, vascular endothelial growth factor; MIA PaCa-2-K, MIA PaCa-2 cells with KAI1 overexpression; MIA PaCa-2-p, empty vector-transfected MIA PaCa-2 cells. 

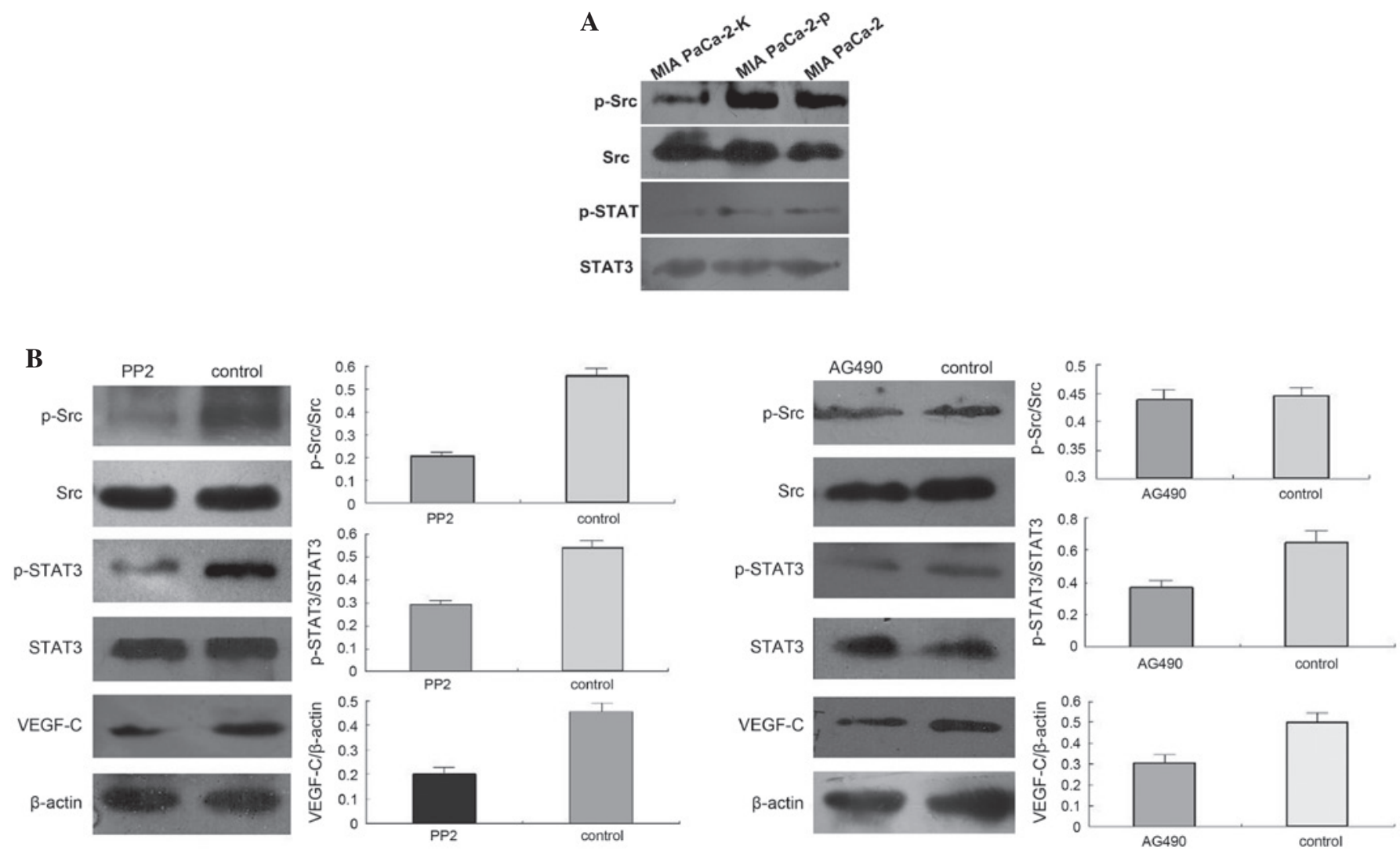

Figure 3. Inhibition of the VEGF-C signaling pathway by KAI1 in MIA PaCa-2 cells. (A) The levels of Src, p-Src, STAT3 and p-STAT3 in MIA PaCa-2, MIA PaCa-2-p and MIA PaCa-2-K cells were evaluated by western blot analysis with $\beta$-actin used as a control. (B) MIA PaCa- 2 cells were treated for $24 \mathrm{~h}$ with $10 \mu \mathrm{mol} / 1$ specific Src inhibitor PP2 or specific STAT3 inhibitor AG490. The effects on the expression of VEGF-C and the abovementioned proteins were assessed by western blot analysis. VEGF, vascular endothelial growth factor; p-STAT3, phosphorylated signal transducer and activator of transcription 3; MIA PaCa-2-K, MIA PaCa-2 cells with KAI1 overexpression; MIA PaCa-2-p, empty vector-transfected MIA PaCa-2 cells.

Tris/HCl, $5 \mathrm{mmol} / \mathrm{l}$ ethylenediaminetetraacetic acid, $50 \mathrm{mmol} / 1$ $\mathrm{NaCl}, 30 \mathrm{mmol} / 1 \mathrm{Na}_{3} \mathrm{PO}_{4}, 50 \mathrm{mmol} / \mathrm{l} \mathrm{NaF}, 0.1 \mathrm{mmol} / \mathrm{l} \mathrm{Na} \mathrm{VO}_{4}$, $1 \mathrm{mmol} / \mathrm{l}$ phenylmethylsulfonyl fluoride, $5 \mathrm{mg} / \mathrm{ml}$ aprotinin and $0.1 \%$ Triton $\mathrm{X}-100 ; \mathrm{pH}$ 7.6) and total protein was quantified using the bicinchoninic acid method (Beijing Solarbio Science \& Technology Co., Ltd., Beijing, China). Equal amounts of protein $(30 \mu \mathrm{g})$ were subjected to $12 \%$ sodium dodecyl sulfate-polyacrylamide gel electrophoresis (Beijing Solarbio Science \& Technology Co., Ltd.) under reducing conditions. The separated proteins were transferred onto polyvinylidene difluoride membranes (EMD Millipore), which were subsequently incubated in blocking buffer [Tris-buffered saline and $0.1 \%$ Tween 20 (TBST) containing 5\% non-fat dry milk) for $2 \mathrm{~h}$ at room temperature. Subsequently, the membranes were incubated at $4{ }^{\circ} \mathrm{C}$ overnight with the following primary antibodies: Polyclonal rabbit anti-KAI1 (1:200 dilution; cat. no. sc-1087; Santa Cruz Biotechnology, Inc., Dallas, TX, USA), polyclonal rabbit anti-VEGF-C (1:500 dilution; cat. no. sc-25783; Santa Cruz Biotechnology, Inc.), rabbit polyclonal anti-Src (1:50 dilution; cat. no. bs-10604R-AP; BIOSS), rabbit polyclonal anti-phospho (p)-Src (1:50 dilution; cat. no. bs-1730R-Bio; BIOSS), rabbit polyclonal anti-STAT3 (1:200 dilution; cat no. sc-7179; Santa Cruz Biotechnology, Inc.), rabbit polyclonal anti-p-STAT3 (1:200 dilution; cat no. sc-71792; Santa Cruz Biotechnology, Inc.) or rabbit polyclonal anti- $\beta$-actin (1:100 dilution; Abcam, Cambridge, UK). After three washes in TBST, the membranes were then incubated with horseradish peroxidase-conjugated secondary antibody rabbit immunoglobulin $\mathrm{G}(1: 2,000$ dilution; cat. no. sc-2749; Santa Cruz Biotechnology, Inc.) for $1 \mathrm{~h}$ at room temperature. Immunoreactive bands were visualized using an Western Lightning Chemiluminescence Reagent Plus kit (Perkin-Elmer Life Science, Boston, MA, USA). The signals were detected using a Las-4000 mini CCD camera (GE Healthcare).

Statistical analysis. Values are expressed as the mean \pm standard deviation from at least three independent experiments. Student's paired $t$-test was used to analyze statistical significance. SPSS 16.0 (SPSS, Inc., Chicago, IL, USA) was used for all statistical analyses. All experiments were repeated three times. $\mathrm{P}<0.05$ was considered to indicate a statistically significant difference between values.

\section{Results}

KAII expression is inversely associated with VEGF-C expression in pancreatic tumor samples. The present study evaluated 28 PC tumor samples by immunohistochemistry (Fig. 1A-C). VEGF-C expression was detected in most tumors with LN metastasis and a negative association was identified between the expression of KAI1 and VEGF-C in tumors with or without node metastasis $(\mathrm{P}<0.05)$ (Fig. 1A-C). Tumors from patients with metastases at NL1 to NL3 and with low KAI1 expression showed high VEGF-C expression $(\mathrm{P}<0.05)$. 
KAIl inhibits VEGF-C expression in PC cell lines. Western blot analysis was used to examine the expression of KAI1 in MIA PaCa-2 and PANC-1 cells. While KAI1 protein was highly expressed in MIA PaCa-2-K and PANC-1-K cells, it was almost undetectable in MIA PaCa-2, MIA PaCa-2-p, PANC-1 and PANC-1-p cells (Fig. 2A). Furthermore, reduced VEGF-C expression was observed in MIA PaCa-2-K and PANC1-K cells compared with that in MIA PaCa-2, MIA PaCa-2-p, PANC1 and PANC1-p cells (Fig. 2A). The reduction of VEGF-C expression was more significant in MIA PaCa-2 cells than that in PANC1 cells (Fig. 2B).

KAII-induced decreases in VEGF-C expression are mediated via Src/STAT3 in MIA PaCa-2 cells. Western blot analysis indicated that Src and STAT3 phosphorylation was markedly decreased in MIA PaCa-2-K cells compared with that in MIA PaCa-2 and MIA PaCa-2-p cells (Fig. 3A). Specific inhibitors of Src (PP2) and STAT3 (AG490) activity were used to determine whether Src and STAT3 activation is required for the KAl1-induced downregulation of VEGF-C expression. Pre-treatment with PP2 efficiently reversed the enhancement of Src and STAT3 phosphorylation and VEGF-C expression (Fig. 3A). Furthermore, pre-treatment with AG490 efficiently reversed the KAI1-induced enhancement of STAT3 phosphorylation and VEGF-C expression, but had no effect on Src phosphorylation (Fig. 3B).

\section{Discussion}

Metastasis is a hallmark of cancer and contributes to $90 \%$ of cancer-associated mortalities (12). As metastastization of cancers largely occurs via the lymphatic system, an understanding of the underlying molecular mechanisms of lymphangiogenesis is of high importance for the development of novel treatment strategies. The present study reported on the specific roles of KAI1 in regulating Src/STAT3/VEGF-C signaling in lymphangiogenesis in PC.

Accumulating evidence has demonstrated that KAI1 is a metastasis-suppressor gene that functions without affecting primary tumorigenicity $(8,13)$. KAI1 signaling pathways and functions are mediated through the activities of a complex molecular network, the expression and activities of which are tightly controlled under physiological conditions. Changes in this delicate balance may trigger a cascade of molecular events that ultimately lead to malignancy. A previous study by our group examined the role of KAI1 in lymphangiogenesis in mice and indicated a negative association with the expression of VEGF-C (9).

VEGF-C secretion by tumor cells can induce the activation of VEGF receptor-3 in the vascular endothelium, thereby inducing the formation of new lymphatic vessels. VEGF-C ha an important stimulatory function in lymphangiogenesis (14), with high VEGF-C expression being significantly associated with LN metastasis, high tumor-nodes-metastasis stage and poor outcome in patients with PC (15). The present study identified a significant negative association between KAI1 and VEGF-C protein expression in pancreatic tumor samples and PC cells. These results confirmed the hypothesis that KAI1 has the potential to suppress the expression of VEGF-C and is its upstream regulator, as posed by a previous study by our group (9). Furthermore, the effect of KAI1 on VEGF-C was more obvious in the highly metastatic MIA PaCa-2 cell line as compared to that in the PANC1 cell line. The result that KAI1 has a more significant inhibitory effect on VEGF-C expression in highly metastatic cells represents a novel characteristic of KAI1. The present study therefore investigated the underlying molecular mechanisms of the effects of KAI1 on VEGF-C to control lymphangiogenesis in the human PC cell lines MIA PaCa-2 and PANC1. Src signaling is an important component of VEGF-C-induced lymphangiogenesis (16). In addition, Src has been indicated to regulate VEGF via STAT3 in human breast cancer as well as in head and neck carcinoma (17). The present study showed that KAI1 inhibits Src and STAT3 phosphorylation as well as VEGF-C expression. However, pre-treatment with the Src inhibitor PP2 efficiently reversed KAI1-induced enhancement of Src and STAT3 phosphorylation and VEGF-C expression. In addition, pre-treatment with the STAT3 inhibitor AG490 efficiently reversed KAI1-induced enhancement of STAT3 phosphorylation and VEGF-C expression, but had no effect on the enhancement of Src phosphorylation in MIA PaCa-2 cells. These results indicated that KAI1 inhibits VEGF-C via the Src/STAT3 signaling pathway. However, density comparison on low-quality blots is not conclusive.

In conclusion, the present study provided the first pre-clinical evidence that KAI1 has the potential to suppress VEGF-C expression in pancreatic tumor samples and the human MIA PaCa-2 and PANC1 PC cell lines. Furthermore, it was demonstrated that KAI1 downregulates VEGF-C expression via the Src/STAT3 signaling pathways in MIA PaCa-2 PC cells. An understanding of the signal transduction mechanisms involved in the KAI1/Src/STAT3/VEGF-C axis may provide a novel approach for the treatment of lymphatic metastasis in PC.

\section{Acknowledgements}

Partial results of the present study have been published in the conference of Gastro 2013 APDW/WCOG in the form of a 'Meeting Abstract'. The authors were Xiao Zhong Guo, Xu Liu, HongYu Li, XiaoDong Shao, ZhongMin Cui, under the title is 'Src/STAT3 signaling pathways are involved in KAI1-reduced VEGF-C down-regulation in PC' pg 881, abstract no. 160. The study was supported by the China Postdoctoral Science Foundation (grant no. 2014M552691).

\section{References}

1. Qian CN, Berghuis B, Tsarfaty G, Bruch M, Kort EJ, Ditlev J, Tsarfaty I, Hudson E, Jackson DG, Petillo D, et al: Preparing the 'soil': The primary tumor induces vasculature reorganization in the sentinel lymph node before the arrival of metastatic cancer cells. Cancer Res 66: 10365-10376, 2006.

2. Stacker SA, Achen MG, Jussila L, Baldwin ME and Alitalo K: Lymphangiogenesis and cancer metastasis. Nat Rev Cancer 2: 573-583, 2002.

3. Stacker SA, Farnsworth RH, Karnezis T, Shayan R, Smith DP, Paavonen K, Davydova N, Caesar C, Inder R, Baldwin ME, et al: Molecular pathways for lymphangiogenesis and their role in human disease. Novartis Found Symp 281: 38-43, 2007.

4. Karpanen T, Wirzenius M, Mäkinen T, Veikkola T, Haisma HJ, Achen MG, Stacker SA, Pytowski B, Ylä-Herttuala S and Alitalo K: Lymphangiogenic growth factor responsiveness is modulated by postnatal lymphatic vessel maturation. Am J Pathol 169: 708-718, 2006. 
5. Skobe M, Hawighorst T, Jackson DG, Prevo R, Janes L, Velasco P, Riccardi L, Alitalo K, Claffey K and Detmar M: Induction of tumor lymphangiogenesis by VEGF-C promotes breast cancer metastasis. Nat Med 7: 192-198, 2001.

6. Hoshida T, Isaka N, Hagendoorn J, di Tomaso E, Chen YL, Pytowski B, Fukumura D, Padera TP and Jain RK: Imaging steps of lymphatic metastasis reveals that vascular endothelial growth factor-C increases metastasis by increasing delivery of cancer cells to lymph nodes: Therapeutic implications. Cancer Res 66: 8065-8075, 2006

7. Liu WM and Zhang XA: KAI1/CD82, a tumor metastasis suppressor. Cancer Lett 240: 183-194, 2006.

8. Miranti CK: Controlling cell surface dynamics and signaling: How CD82/KAI1 suppresses metastasis. Cell Signal 21: 196-211, 2009.

9. Liu X, Guo XZ, Li HY, Chen J, Ren LN and Wu CY: KAI1 inhibits lymphangiogenesis and lymphatic metastasis of pancreatic cancer in vivo. Hepatobiliary Pancreat Dis Int 13: 87-92, 2014.

10. Cheng P, Jin G, Hu X, Shi M, Zhang Y, Liu R, Zhou Y, Shao C, Zheng J and Zhu M: Analysis of tumor-induced lymphangiogenesis and lymphatic vessel invasion of pancreatic carcinoma in the peripheral nerve plexus. Cancer Sci 103: 1756-1763, 2012.

11. Japan Pancreas Society. Classification of pancreatic carcinoma. 3rd English edn. Kanehara, Tokyo, 2003.
12. Yan J, Yang Q and Huang Q: Metastasis suppressor genes Histol Histopathol 28: 285-292, 2013.

13. Liu X, Guo XZ, Zhang WW, Lu ZZ, Zhang QW, Duan HF and Wang LS: KAI1 inhibits HGF-induced invasion of pancreatic cancer by sphingosine kinase activity. Hepatobiliary Pancreat Dis Int 10: 201-208, 2011.

14. Matsumoto M, Roufail S, Inder R, Caesar C, Karnezis T, Shayan R, Farnsworth RH, Sato T, Achen MG, Mann GB and Stacker SA: Signaling for lymphangiogenesis via VEGFR-3 is required for the early events of metastasis. Clin Exp Metastasis 30: 819-832, 2013.

15. Kurahara H, Takao S, Maemura K, Shinchi H, Natsugoe S and Aikou T: Impact of vascular endothelial growth factor-C and -D expression in human pancreatic cancer: Its relationship to lymph node metastasis. Clin Cancer Res 10: 8413-8420, 2004.

16. Ischenko I, Seeliger H, Camaj P, Kleespies A, Guba M, Eichhorn ME, Jauch KW and Bruns CJ: Src tyrosine kinase inhibition suppresses lymphangiogenesis in vitro and in vivo. Curr Cancer Drug Targets 10: 546-553, 2010.

17. Niu G, Wright KL, Huang M, Song L, Haura E, Turkson J, Zhang S, Wang T, Sinibaldi D, Coppola D, et al: Constitutive Stat 3 activity up-regulates VEGF expression and tumor angiogenesis. Oncogene 21: 2000-2008, 2002. 